ISSN = 1980-993X - doi:10.4136/1980-993X
www.ambi-agua.net
E-mail: ambi-agua@agro.unitau.br
Tel.: (12) 3625-4212

\title{
Interpolação geoespacial da evapotranspiração de referência (ETo) em regiões com escassez de dados: estudo de caso no Sul de Minas Gerais, Brasil
}

\author{
(http://dx.doi.org/10.4136/ambi-agua.763)
}

\author{
Mateus Ricardo Nogueira Vilanova ${ }^{1}$; Silvio J. C. Simões ${ }^{2}$; Isabel C. B. Trannin ${ }^{2}$ \\ ${ }^{\mathbf{1}}$ Faculdade de Engenharia de Guaratinguetá-FEG, (UNESP), Programa de Pós-graduação em Engenharia \\ Mecânica (Transmissão e Conversão de Energia) \\ e-mail: mathidr@yahoo.com.br, \\ ${ }^{2}$ Faculdade de Engenharia de Guaratinguetá-FEG, (UNESP), Departamento de Engenharia Civil, \\ e-mails: silvio.jorge.simoes@gmail.com, isatrannin@uol.com.br
}

\section{RESUMO}

A evapotranspiração de referência é uma importante variável hidrometeorológica, cuja medição é escassa em grande parte do território brasileiro, exigindo a aplicação de métodos e técnicas alternativas para sua quantificação. Nesse sentido, o presente trabalho avalia o processo de espacialização da evapotranspiração de referência por meio do método geoestatístico de krigagem, em regiões com pouca disponibilidade de estações e dados hidrometeorológicos. A evapotranspiração de referência média mensal foi calculada pela equação de Penman-Monteith-FAO, a partir de dados de três estações meteorológicas localizadas no Sul de Minas Gerais (Itajubá, Lavras e Poços de Caldas) e, posteriormente, interpolada por krigagem ordinária pontual, utilizando a abordagem "calcular e interpolar". Os dados hidrometeorológicos de uma quarta estação (Três Corações), localizada dentro da área de interpolação, foram utilizados para validar as evapotranspirações de referência interpoladas espacialmente. Devido à reduzida quantidade de estações e conseqüente inviabilidade de se realizarem análises variográficas, a comparação entre as ETo calculadas e interpoladas para a estação Três Corações foi realizada por meio do coeficiente de correlação (r), índice de concordância (d), erro de viés médio (MBE), raiz do erro médio quadrático (RMSE) e t-test. Os resultados desta comparação indicam que o procedimento de espacialização por krigagem, mesmo dispondo de poucas estações, permite interpolar de forma satisfatória a evapotranspiração de referência, sendo uma importante ferramenta para aplicações agrícolas e hidrológicas em regiões pouco monitoradas.

Palavras-chave: hidrometeorologia, método de Penman-Monteith-FAO;krigagem, espacialização.

\section{Geospatial interpolation of reference evapotranspiration (ETo) in areas with scarce data: case study in the South of Minas Gerais, Brazil}

\begin{abstract}
The reference evapotranspiration is an important hydrometeorological variable; its measurement is scarce in large portions of the Brazilian territory, what demands the search for alternative methods and techniques for its quantification. In this sense, the present work investigated a method for the spatialization of the reference evapotranspiration using the geostatistical method of kriging, in regions with limited data and hydrometeorological stations. The monthly average reference evapotranspiration was calculated by the PenmanMonteith-FAO equation, based on data from three weather stations located in southern Minas
\end{abstract}


Gerais (Itajubá, Lavras and Poços de Caldas), and subsequently interpolated by ordinary point kriging using the approach "calculate and interpolate." The meteorological data for a fourth station (Três Corações) located within the area of interpolation were used to validate the reference evapotranspiration interpolated spatially. Due to the reduced number of stations and the consequent impossibility of carrying variographic analyzes, correlation coefficient (r), index of agreement (d), medium bias error (MBE), root mean square error (RMSE) and t-test were used for comparison between the calculated and interpolated reference evapotranspiration for the Três Corações station. The results of this comparison indicated that the spatial kriging procedure, even using a few stations, allows to interpolate satisfactorily the reference evapotranspiration, therefore, it is an important tool for agricultural and hydrological applications in regions with lack of data.

Keywords: hydrometeorology, Penman-Monteith-FAO, kriging;spatialization.

\section{INTRODUÇÃO}

A evapotranspiração de referência $\left(\mathrm{ET}_{\mathrm{o}}\right)$ é uma importante variável hidrometeorológica utilizada na agricultura e gerenciamento de recursos hídricos, sendo fundamental também para modelagem hidrológica distribuída (Zhang et al., 2010). Por definição, a $\mathrm{ET}_{\mathrm{o}}$ corresponde à evapotranspiração de uma cultura hipotética, cobrindo totalmente o solo, em crescimento ativo, sob condições ótimas de desenvolvimento (sem restrições hídricas e nutricionais), com altura média de $0,12 \mathrm{~m}$, albedo de 0,23 e resistência da superfície de 70 s.m ${ }^{-1}$ (Bernardo et al., 2008). Apesar da importância da $\mathrm{ET}_{\mathrm{o}}$ para a estudos e gestão de bacias hidrográficas, especialmente em áreas irrigadas e florestais, a medição da evapotranspiração é cara e escassa, demandando equipamentos e instrumentos específicos (Silva et al., 2010). Diante desse fato, várias pesquisas foram desenvolvidas, resultando em equações e métodos práticos para a determinação indireta da $\mathrm{ET}_{\mathrm{o}}$.

Dentre os métodos de estimativa da $\mathrm{ET}_{\mathrm{o}}$ amplamente utilizados na atualidade, destaca-se o Penman-Monteith-FAO, de base física, recomendado e difundido pela Organização das Nações Unidas para Agricultura e Alimentação (FAO) (Allen et al., 1998). Segundo Yin et al. (2008), o modelo Penman-Monteith-FAO, que incorpora aspectos termodinâmicos e aerodinâmicos em seu equacionamento, produz resultados satisfatoriamente precisos tanto em climas úmidos quanto áridos, sendo aceito e aplicado com êxito em todo mundo (Er-Raki et al., 2010).

As aplicações práticas dos dados de evapotranspiração - seja para o planejamento de irrigação, modelagem hidrológica e ambiental, dentre outras - exigem quase sempre que estes sejam avaliados de forma distribuída espacialmente (Mcvicar et al., 2007), residindo aí a importância da elaboração de mapas de $\mathrm{ET}_{\mathrm{o}}$.

A espacialização de dados hidrológicos, climáticos e ambientais pode ser feita a partir de métodos geoestatísticos, sendo a krigagem comumente utilizada (Mardikis et al., 2005; Bargaoui e Chebbi, 2009). A krigagem utiliza a "[...] dependência espacial entre amostras vizinhas, expressa no semivariograma, para estimar valores em qualquer posição dentro do espaço analisado ao qual o modelo do semivariograma foi ajustado, sem tendência e com variância mínima." (Motomiya et al., 2006). Utilizada em diversas áreas do conhecimento, a krigagem assume que a distância ou a diferença entre os pontos de amostragem refletem uma correlação espacial que pode ser utilizada para explicar sua variação em uma superfície (Childs, 2004). Trabalhos como os de Mello et al. (2003) e Alves et al. (2008) indicam desempenho superior da krigagem em relação a outros métodos geoestatísticos, como o do inverso da potência da distância. 
Zhang et al. (2010) e Mardikis et al. (2005) apresentam duas abordagens para o mapeamento da $\mathrm{ET}_{\mathrm{o}}$. Na primeira, denominada "calcular e interpolar", as $\mathrm{ET}_{\mathrm{o}}$ são calculadas para as estações meteorológicas de referência e, posteriormente,espacializadas. Na abordagem "interpolar e calcular", as variáveis meteorológicos utilizados no equacionamento da $\mathrm{ET}_{\mathrm{o}}$ são espacializados, e a $\mathrm{ET}_{\mathrm{o}}$ posteriormente calculada para as áreas de interesse, o que torna tal abordagem mais laboriosa que a "calcular e interpolar".

Diante do exposto, o trabalho tem por objetivo avaliar a qualidade dos valores de $\mathrm{ET}_{\mathrm{o}}$ médios mensais interpolados geospacialmente a partir do processo de krigagem, com pequena disponibilidade de dados/estações hidrometeorológicas - no caso estudado, foram consideradas apenas três estações, localizadas no sul de Minas Gerais. Uma quarta estação, localizada dentro da área de interpolação, foi utilizada como referência para validação da interpolação, por meio da comparação entre os valores de $\mathrm{ET}_{\mathrm{o}}$ calculados com dados dessa estação, e aqueles interpolados a partir das demais. A pequena quantidade de estações empregadas na interpolação inviabiliza a análise variográfica, tipicamente utilizada para se avaliar os resultados da krigagem, exigindo que a análise da qualidade da interpolação seja realizada por meio do coeficiente de correlação (r), índice de concordância (d), erro médio de viés (MBE), raiz do erro médio quadrático (RMSE) e t-test.

\section{MATERIAL E MÉTODOS}

O sul do Estado de Minas Gerais foi considerado como área de estudo, sendo selecionadas as estações meteorológicas dos municípios de Itajubá, Lavras, Poços de Caldas e Três Corações, cujos dados de temperatura mínima, temperatura máxima, umidade relativa do ar, velocidade média do vento, insolação e radiação foram obtidos na base do Grupo de Agrometeorologia da Organização das Nações Unidas para Agricultura e Alimentação (FAO). A Tabela 1 apresenta as coordenadas das estações selecionadas.

Tabela 1. Estações meteorológicas dos municípios do Sul de Minas Gerais empregadas no estudo

\begin{tabular}{l|lc|cc}
\hline \multicolumn{5}{c}{ Coordenadas aproximadas das estações utilizadas (WGS84) } \\
\hline \multirow{2}{*}{ Estação } & \multicolumn{2}{|c}{ Geográficas } & \multicolumn{2}{c}{ UTM (23 K) } \\
\cline { 2 - 5 } & ${\text { Latitude }\left[{ }^{\circ}\right]}^{\circ}$ & Longitude [ $\left.{ }^{\circ}\right]$ & E [m] & N [m] \\
\hline Três Corações (TC) & $21^{\circ} 42^{\prime} 04^{\prime \prime} \mathrm{S}$ & $45^{\circ} 14^{\prime} 59^{\prime \prime} \mathrm{O}$ & $474.169,73$ & $7.600 .234,43$ \\
Itajubá (IT) & $22^{\circ} 25^{\prime} 43 " \mathrm{~S}$ & $45^{\circ} 27^{\prime} 11^{\prime \prime} \mathrm{O}$ & $453.376,70$ & $7.519 .660,22$ \\
Lavras (LV) & $21^{\circ} 13^{\prime} 48 " \mathrm{~S}$ & $45^{\circ} 00^{\prime} 06^{\prime \prime} \mathrm{O}$ & $499.827,05$ & $7.652 .397,28$ \\
Poços de Caldas (PC) & $21^{\circ} 50^{\prime} 58^{\prime \prime} \mathrm{S}$ & $46^{\circ} 33^{\prime} 45^{\prime \prime} \mathrm{O}$ & $338.533,17$ & $7.583 .017,74$ \\
\hline
\end{tabular}

Fonte: FAO ( 2006).

Os critérios adotados para a seleção das estações foram a proximidade geográfica (distância inferior a $200 \mathrm{~km}$ ) e a inexistência de feições topográficas significativas entre elas, buscando obter uma maior homogeneidade dos parâmetros meteorológicos utilizados no cálculo da $\mathrm{ET}_{\mathrm{o}}$, conforme recomenda o trabalho de McVicar et al. (2007). São consideradas significativas as feições topográficas que atuam sobre as condições hidrometeorológicas e hidroclimáticas regionais, ao contrário de pequenas unidades de relevo que influenciam tais condições de forma local (microclima). Um exemplo de feição significativa é a Serra da 
VILANOVA, M. R. N.; SIMÕES, S. J. C.; TRANNIN, I. C, B. Interpolação geoespacial da evapotranspiração de referência $\left(E_{\mathrm{o}}\right)$ em regiões com escassez de dados: estudo de caso no Sul de Minas Gerais. Ambi-Agua, Taubaté, v. 7, n. 2, p. 179-194, 2012. (http://dx.doi.org/10.4136/ambi-agua.763)

Mantiqueira, que tem papel fundamental no estabelecimento de condições meteorológicas distintas entre o Sul de Minas Gerais e o Vale do Rio Paraíba do Sul, apesar da proximidade geográfica das duas regiões. Todas as estações possuem dados históricos (médias mensais) de no mínimo 15 anos de observação (1975 a 2000), sendo estes obtidos com auxílio do aplicativo Climwat 2.0 (FAO, 2006), fornecido pela Organização das Nações Unidas para Agricultura e Alimentação (FAO). Posteriormente, as ET $_{0}$ médias mensais foram calculadas pelo método de Penman-Monteith-FAO, pela Equação [1].

$$
E T_{O}=\frac{0,408 \cdot \Delta \cdot(R n-G)+\gamma \cdot \frac{900}{T+273} \cdot U_{2} \cdot\left(e_{s}-e_{a}\right)}{\Delta+\gamma \cdot\left(1+0,34 \cdot U_{2}\right)}
$$

em que:

$\mathrm{ET}_{\mathrm{o}} \quad$ evapotranspiração de referência $\left[\mathrm{mm}_{\mathrm{d}} \mathrm{d}^{-1}\right]$

$\mathrm{Rn} \quad$ saldo de radiação à superfície $\left[\mathrm{MJ} \cdot \mathrm{m}^{-2} \cdot \mathrm{d}^{-1}\right]$;

$\mathrm{G} \quad$ fluxo de calor no solo $\left[\mathrm{MJ} \cdot \mathrm{m}^{-2} \cdot \mathrm{d}^{-1}\right]$;

$\mathrm{T}$ temperatura do ar $\left[{ }^{\circ} \mathrm{C}\right]$;

$\mathrm{U}_{2}$ velocidade do vento [m.s ${ }^{-1}$;

$\mathrm{e}_{\mathrm{s}} \quad$ pressão de saturação de vapor d'água no ar [kPa];

$\mathrm{e}_{\mathrm{a}} \quad$ pressão de vapor d'água no ar $[\mathrm{kPa}]$

$\left(\mathrm{e}_{\mathrm{s}}-\mathrm{e}_{\mathrm{a}}\right)$ déficit de pressão de vapor d'água no ar [kPa];

$\Delta \quad$ declividade da curva de pressão de saturação de vapor d'água no $\operatorname{ar}\left[\mathrm{kPa}^{\circ}{ }^{\circ} \mathrm{C}^{-1}\right]$;

$\gamma \quad$ constante psicrométrica $\left[\mathrm{kPa} .{ }^{0} \mathrm{C}^{-1}\right]$.

As Tabelas 2 a 5 apresentam os dados meteorológicos médios mensais de cada estação, bem como as $\mathrm{ET}_{\mathrm{o}}$ calculadas.

Tabela 2. Dados climatológicos para a localidade de Três Corações.

\begin{tabular}{|c|c|c|c|c|c|c|c|}
\hline \multicolumn{8}{|c|}{ TRÊS CORAÇÕES (TC) } \\
\hline Mês & $\begin{array}{c}\text { Temperatura } \\
\text { mínima } \\
{ }^{\circ} \mathbf{C}\end{array}$ & $\begin{array}{c}\text { Temperatura } \\
\text { máxima } \\
{ }^{\circ} \mathbf{C}\end{array}$ & $\begin{array}{c}\text { Umidade } \\
\text { do ar } \\
\%\end{array}$ & $\begin{array}{c}\text { Velocidade } \\
\text { do vento } \\
\text { km.d }\end{array}$ & $\begin{array}{c}\text { Insolação } \\
\mathbf{h}\end{array}$ & $\begin{array}{c}\text { Radiação } \\
\text { MJ.(m².d })^{-1}\end{array}$ & $\underset{\text { mm.d }}{\text { ETo }}$ \\
\hline Jan & 17,5 & 29,1 & 79,0 & 61,0 & 6,7 & 21,2 & 4,3 \\
\hline Fev & 17,6 & 29,1 & 81,0 & 78,0 & 5,9 & 19,2 & 4,0 \\
\hline Mar & 16,5 & 28,0 & 81,0 & 86,0 & 5,9 & 17,8 & 3,6 \\
\hline Abr & 14,0 & 26,7 & 80,0 & 86,0 & 6,3 & 16,0 & 3,1 \\
\hline Mai & 10,6 & 24,9 & 80,0 & 69,0 & 6,5 & 14,0 & 2,4 \\
\hline Jun & 8,0 & 23,8 & 77,0 & 69,0 & 6,4 & 12,8 & 2,1 \\
\hline Jul & 8,2 & 23,9 & 75,0 & 69,0 & 6,8 & 13,8 & 2,1 \\
\hline Ago & 9,5 & 26,5 & 69,0 & 86,0 & 7,2 & 16,2 & 2,9 \\
\hline Set & 12,3 & 27,5 & 71,0 & 104,0 & 5,6 & 16,5 & 3,3 \\
\hline Out & 14,6 & 27,5 & 73,0 & 112,0 & 5,3 & 17,8 & 3,7 \\
\hline Nov & 15,8 & 27,8 & 76,0 & 112,0 & 5,4 & 18,9 & 4,0 \\
\hline Dez & 16,9 & 27,8 & 80,0 & 104,0 & 4,8 & 18,2 & 3,8 \\
\hline
\end{tabular}

Fonte: FAO (2006). 
VILANOVA, M. R. N.; SIMÕES, S. J. C.; TRANNIN, I. C, B. Interpolação geoespacial da evapotranspiração de referência $\left(\mathrm{ET}_{\mathrm{o}}\right)$ em regiões com escassez de dados: estudo de caso no Sul de Minas Gerais. Ambi-Agua, Taubaté, v. 7, n. 2, p. 179-194, 2012. (http://dx.doi.org/10.4136/ambi-agua.763)

Tabela 3. Dados climatológicos para a localidadede Itajubá.

\begin{tabular}{|c|c|c|c|c|c|c|c|}
\hline \multicolumn{8}{|c|}{ Itajubá (IT) } \\
\hline Mês & $\begin{array}{c}\text { Temperatura } \\
\text { mínima } \\
{ }^{\circ} \mathrm{C}\end{array}$ & $\begin{array}{c}\text { Temperatura } \\
\text { máxima } \\
{ }^{\circ} \mathbf{C}\end{array}$ & $\begin{array}{c}\text { Umidade } \\
\text { do ar } \\
\%\end{array}$ & $\begin{array}{l}\text { Velocidade } \\
\text { do vento } \\
\text { km.d }\end{array}$ & $\begin{array}{c}\text { Insolação } \\
\text { H }\end{array}$ & 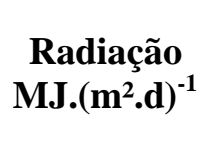 & $\begin{array}{c}\text { ETo } \\
\text { mm.d }^{-1}\end{array}$ \\
\hline Jan & 17,3 & 29,0 & 79,0 & 61,0 & 6,8 & 21,4 & 4,3 \\
\hline $\mathrm{Fev}$ & 17,3 & 29,0 & 80,0 & 78,0 & 5,8 & 19,0 & 3,9 \\
\hline Mar & 16,5 & 28,7 & 79,0 & 86,0 & 6,0 & 17,9 & 3,7 \\
\hline Abr & 14,0 & 27,1 & 78,0 & 86,0 & 6,3 & 15,9 & 3,1 \\
\hline Mai & 10,7 & 25,2 & 78,0 & 69,0 & 6,5 & 13,9 & 2,4 \\
\hline Jun & 8,8 & 24,2 & 76,0 & 69,0 & 6,5 & 12,8 & 2,1 \\
\hline Jul & 8,1 & 24,3 & 72,0 & 69,0 & 6,8 & 13,6 & 2,2 \\
\hline Ago & 9,5 & 26,5 & 68,0 & 86,0 & 7,1 & 16,0 & 2,9 \\
\hline Set & 12,1 & 27,3 & 68,0 & 104,0 & 5,7 & 16,5 & 3,4 \\
\hline Out & 14,5 & 27,8 & 71,0 & 112,0 & 5,3 & 17,7 & 3,8 \\
\hline Nov & 15,6 & 28,1 & 75,0 & 112,0 & 5,5 & 19,1 & 4,0 \\
\hline Dez & 16,7 & 28,2 & 78,0 & 104,0 & 4,9 & 18,4 & 3,9 \\
\hline
\end{tabular}

Fonte: FAO (2006).

Tabela 4. Dados climatológicos para a localidadede Lavras.

\begin{tabular}{|c|c|c|c|c|c|c|c|}
\hline \multicolumn{8}{|c|}{ Lavras (LV) } \\
\hline Mês & $\begin{array}{c}\text { Temperatura } \\
\text { mínima } \\
{ }^{\circ} \mathrm{C}\end{array}$ & $\begin{array}{c}\text { Temperatura } \\
\text { máxima } \\
{ }^{\circ} \mathbf{C}\end{array}$ & $\begin{array}{c}\text { Umidade } \\
\text { do ar } \\
\%\end{array}$ & $\begin{array}{c}\text { Velocidade } \\
\text { do vento } \\
\text { km.d }\end{array}$ & $\begin{array}{c}\text { Insolação } \\
\mathbf{h}\end{array}$ & 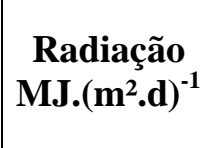 & $\underset{\text { mm.d }}{\text { ETo }}$ \\
\hline Jan & 17,7 & 27,8 & 84,0 & 61,0 & 5,6 & 19,4 & 3,9 \\
\hline Fev & 17,9 & 28,4 & 82,0 & 78,0 & 5,7 & 19,0 & 3,9 \\
\hline Mar & 17,3 & 27,0 & 84,0 & 86,0 & 6,0 & 18,0 & 3,6 \\
\hline Abr & 15,4 & 25,4 & 78,0 & 86,0 & 6,5 & 16,3 & 3,1 \\
\hline Mai & 12,7 & 24,7 & 77,0 & 69,0 & 6,5 & 14,1 & 2,4 \\
\hline Jun & 11,1 & 23,9 & 76,0 & 69,0 & 6,1 & 12,6 & 2,1 \\
\hline Jul & 10,4 & 23,7 & 73,0 & 69,0 & 6,6 & 13,6 & 2,2 \\
\hline Ago & 11,7 & 25,7 & 67,0 & 86,0 & 7,2 & 16,3 & 2,9 \\
\hline Set & 13,6 & 25,4 & 70,0 & 104,0 & 5,9 & 16,9 & 3,3 \\
\hline Out & 15,6 & 27,2 & 74,0 & 112,0 & 5,7 & 18,4 & 3,8 \\
\hline Nov & 16,6 & 27,2 & 77,0 & 112,0 & 5,5 & 19,1 & 3,9 \\
\hline Dez & 17,3 & 27,3 & 84,0 & 104,0 & 4,8 & 18,2 & 3,7 \\
\hline
\end{tabular}

Fonte: FAO (2006). 
VILANOVA, M. R. N.; SIMÕES, S. J. C.; TRANNIN, I. C, B. Interpolação geoespacial da evapotranspiração de referência $\left(\mathrm{ET}_{\mathrm{o}}\right)$ em regiões com escassez de dados: estudo de caso no Sul de Minas Gerais. Ambi-Agua, Taubaté, v. 7, n. 2, p. 179-194, 2012. (http://dx.doi.org/10.4136/ambi-agua.763)

Tabela 5. Dados climatológicos para a localidadede Poços de Caldas

\begin{tabular}{|c|c|c|c|c|c|c|c|}
\hline \multicolumn{8}{|c|}{ Poços de Caldas (PC) } \\
\hline Mês & $\begin{array}{c}\text { Temperatura } \\
\text { mínima } \\
{ }^{\circ} \mathbf{C}\end{array}$ & $\begin{array}{c}\text { Temperatura } \\
\text { máxima } \\
{ }^{\circ} \mathbf{C}\end{array}$ & $\begin{array}{c}\text { Umidade } \\
\text { do ar } \\
\%\end{array}$ & $\begin{array}{l}\text { Velocidade } \\
\text { do vento } \\
\text { km.d }^{-1}\end{array}$ & $\begin{array}{c}\text { Insolação } \\
\mathbf{h}\end{array}$ & 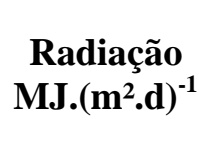 & $\begin{array}{c}\text { ETo } \\
\text { mm.d }^{-1}\end{array}$ \\
\hline Jan & 16,2 & 25,5 & 83,0 & 121,0 & 4,9 & 18,4 & 3,7 \\
\hline Fev & 16,2 & 25,9 & 82,0 & 121,0 & 5,1 & 18,0 & 3,6 \\
\hline Mar & 15,4 & 25,6 & 83,0 & 121,0 & 6,0 & 17,8 & 3,5 \\
\hline Abr & 12,7 & 24,3 & 81,0 & 95,0 & 6,7 & 16,5 & 3,0 \\
\hline Mai & 9,2 & 22,5 & 80,0 & 121,0 & 6,7 & 14,2 & 2,4 \\
\hline Jun & 7,5 & 21,4 & 78,0 & 112,0 & 6,8 & 13,2 & 2,1 \\
\hline Jul & 7,4 & 21,6 & 76,0 & 112,0 & 6,8 & 13,7 & 2,2 \\
\hline Ago & 8,9 & 23,8 & 70,0 & 130,0 & 7,3 & 16,3 & 2,9 \\
\hline Set & 11,4 & 24,8 & 71,0 & 130,0 & 6,2 & 17,3 & 3,4 \\
\hline Out & 13,7 & 25,3 & 75,0 & 130,0 & 6,0 & 18,9 & 3,7 \\
\hline Nov & 14,6 & 25,2 & 79,0 & 121,0 & 5,8 & 19,5 & 3,8 \\
\hline Dez & 15,6 & 25,2 & 82,0 & 121,0 & 5,3 & 19,1 & 3,8 \\
\hline
\end{tabular}

Fonte: FAO (2006).

A estação do município de Três Corações foi adotada para avaliação do método de interpolação geoespacial da $\mathrm{ET}_{0}$, devido ao fato desta se encontrar na região interior ao triângulo cujos vértices são posicionados, geograficamente, sobre as demais estações. Foi realizada uma análise de correlação, efetuando regressão linear entre os dados de $\mathrm{ET}_{\mathrm{o}}$ calculados para a estação de Três Corações e para as estações utilizadas na interpolação.

Realizou-se então o processo de espacialização das $\mathrm{ET}_{\mathrm{o}}$ médias mensais das estações Itajubá, Lavras e Poços de Caldas, por meio de krigagem ordinária pontual. As coordenadas das estações (Tabela 1) foram relacionadas com as respectivas $\mathrm{ET}_{\mathrm{o}}$ mensais (Tabelas 3 a 5). $\mathrm{As}_{\mathrm{ET}}$ foram interpoladas utilizando sistema de informação geográfica, dando origem aos 12 mapas de $\mathrm{ET}_{\mathrm{o}}$ médias mensais.

A krigagem é um método de interpolação semelhante ao da média móvel ponderada, diferindo deste no cálculo dos pesos, definidos a partir de uma análise espacial baseada no semivariograma experimental (Câmara e Medeiros, 1998).

Segundo Burrough e McDonnell (1998), o valor de uma variável espacializada Z é fornecido pela Equação [2]:

$$
Z(x)=m(x)+\varepsilon^{\prime}(x)+\varepsilon^{\prime \prime}
$$

em que: $\begin{array}{ll}Z(x) & \text { valor da variável Z, na posição x; } \\ X & \text { posição em uma, duas ou três dimensões; } \\ \mathrm{m}(\mathrm{x}) & \text { função determinística que descreve a componente estrutural de } \mathrm{Z} \text { em x; } \\ \varepsilon^{\prime} & \text { termo estocástico que varia localmente e depende espacialmente de m(x); } \\ \varepsilon^{\prime \prime} & \text { ruído aleatório não correlacionado, com distribuição normal, média zero e } \\ \text { variância } \square 2 .\end{array}$ 
Os variogramas e semivariogramas expressam a variação espacial de uma variável, sendo ferramentas fundamentais para a krigagem, segundo Câmara e Medeiros (1998) e Guedes (2008). O variograma de uma amostra pode ser expresso pela Equação [3]:

$$
\hat{2 \gamma(h)}=\frac{1}{N(h)} \cdot \sum_{i=1}^{N(h)}\left[z\left(x_{i}\right)-z\left(x_{i}+h\right)\right]^{2}
$$

em que:

$\hat{2} \hat{\gamma}(z h) \quad$ variograma estimado;

$\mathrm{h} \quad$ vetor distância entre dois pontos de amostragem;

$\mathrm{N}(\mathrm{h}) \quad$ número de pares de valores medidos separados por $\mathrm{h}, \mathrm{z}(\mathrm{xi}) \mathrm{e} \mathrm{z}(\mathrm{xi}+\mathrm{h})$;

$\mathrm{z}(\mathrm{xi})$ e $\mathrm{z}(\mathrm{xi}+\mathrm{h})$ valores da i-ésima observação da variável regionalizada, coletadas nos pontos xi e $(x i+h)$.

O estimador de krigagem ordinário, por sua vez, é dado pela Equação [4]:

$$
\mathrm{Z}_{\mathrm{x}_{\mathrm{o}}}^{*}=\sum_{\mathrm{i}=1}^{\mathrm{n}} \lambda_{\mathrm{i}} \cdot \mathrm{Z}\left(\mathrm{x}_{\mathrm{i}}\right)
$$

em que:

$\mathrm{Z}_{\mathrm{x}_{\mathrm{o}}}^{*} \quad$ estimador de krigagem ordinária para o ponto xo;

$\lambda_{\mathrm{i}} \quad$ i-ésimo peso;

Z(xi) valor da i-ésima observação da variável regionalizada, coletada nos pontos xi.

O equacionamento do estimador de krigagem ordinária pressupõe um estimador não tendencioso. Além disso, as Equações [5] e [6] devem ser atendidas (Câmara; Medeiros, 1998):

$$
\begin{aligned}
& \sum_{i=1}^{n} \lambda_{i}=1 \\
& \lambda_{0}=0
\end{aligned}
$$

O sistema de krigagem ordinária apresentado por Journel (1989) é representado pelo seguinte sistema de equações:

$$
\left\{\begin{array}{l}
\sum_{j=1}^{n} \lambda_{j} \cdot C\left(x_{i}, x_{j}\right)-\alpha=C\left(x_{i}, x_{0}\right) \quad \text { parai }=1, \ldots, n \\
\sum_{j=1}^{n} \lambda_{j}=1
\end{array}\right.
$$

em que:

$C\left(x_{i}, x_{j}\right)$ e $C\left(x_{i}, x_{0}\right)$ são semivariâncias entre os pontos $x_{i}$ e $x_{j}$ e entre os pontos $x_{i}$ e $x_{0}$, respectivamente;

$\alpha \quad$ multiplicador Lagrangeano necessário para a minimização da variância do erro. 
Ainda segundo Journel (1989), o sistema de krigagem ordinária é resolvido minimizando-se a variância do erro, ao mesmo tempo em que é atendida a Equação [5].

A comparação dos valores de ETO médias mensais calculados (a partir dos dados meteorológicos da estação) e interpolados por krigagem para a estação Três Corações foi efetuada por meio do t-test (Decoursey, 2003; Ross, 2004; Toutenburg, 2002), do coeficiente de correlação (r) e índice de concordância (d) propostos por Willmott (1981), além do erro médio de viés (MBE) e raiz do erro médio quadrático (RMSE) sugeridos por Jacovides e Kontoyiannis (1995). Tais indicadores são calculados pelas Equações [8] a [11].

$$
\begin{gathered}
r=\frac{\sum_{i=1}^{n}\left(E T_{O O i}-\overline{E T_{O O i}}\right) \cdot\left(E T_{O E i}-\overline{E T_{O E i}}\right)}{\sqrt{\sum_{i=1}^{n}\left(E T_{O O i}-\overline{E T_{O O i}}\right)^{2} \cdot \sum_{i=1}^{n}\left(E T_{O E i}-\overline{E T_{O E i}}\right)^{2}}} \\
\mathrm{~d}=1-\left[\frac{\sum_{\mathrm{i}=1}^{\mathrm{n}}\left(\mathrm{ET}_{\mathrm{OEi}}-\mathrm{ET}_{\mathrm{OOi}}\right)^{2}}{\sum_{\mathrm{i}=1}^{\mathrm{n}}\left(\left|\mathrm{ET}_{\mathrm{OEi}}-\overline{\mathrm{ET}_{\mathrm{OOi}}}\right|+\left|\mathrm{ET}_{\mathrm{OOi}}-\overline{\mathrm{ET}_{\mathrm{OOi}}}\right|\right)^{2}}\right] \\
\mathrm{MBE}=\frac{1}{\mathrm{n}} \cdot \sum_{\mathrm{i}=1}^{\mathrm{n}}\left(\mathrm{ET}_{\mathrm{OEi}}-\mathrm{ET}_{\mathrm{OOi}}\right) \\
\mathrm{RMSE}=\sqrt{\frac{1}{\mathrm{n}} \cdot \sum_{\mathrm{i}=1}^{\mathrm{n}}\left(\mathrm{ET}_{\mathrm{OEi}}-\mathrm{ET}_{\mathrm{OOi}}\right)^{2}}
\end{gathered}
$$

em que:
r $\quad$ coeficiente de correlação [1];
d índice de concordância [1];
RMSE raiz do erro médio quadrático $\left[\mathrm{mm}^{-1} \mathrm{~d}^{-1}\right.$;
MBE erro médio de viés $\left[\mathrm{mm}^{-1} \mathrm{~d}^{-1}\right.$;
ET $_{\mathrm{OEi}}$ evapotranspiração de referência estimada (interpolada) $\left[\mathrm{mm}_{\mathrm{d}} \mathrm{d}^{-1}\right]$;
ET $_{\text {OOi }}$ evapotranspiração de referência observada (calculada) $\left[\mathrm{mm}_{\mathrm{d}} \mathrm{d}^{-1}\right]$;

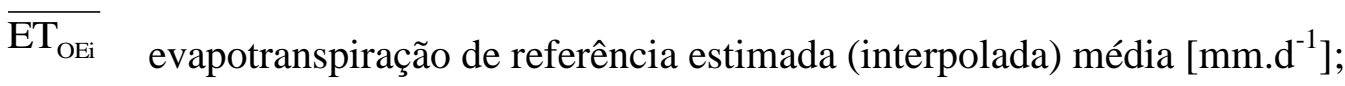
$\overline{\mathrm{ET}_{\mathrm{OOi}}}$ evapotranspiração de referência observada (calculada) média $\left[\mathrm{mm} \cdot \mathrm{d}^{-1}\right]$.

\section{RESULTADOS E DISCUSSÃO}

As Figuras 1, 2 e 3 apresentam a regressão linear e os coeficientes de determinação $\left(\mathrm{R}^{2}\right)$ entre as ETo médias mensais calculadas da estação de referência (Três Corações) e das estações utilizadas na interpolação: 
VILANOVA, M. R. N.; SIMÕES, S. J. C.; TRANNIN, I. C, B. Interpolação geoespacial da evapotranspiração de referência $\left(\mathrm{ET}_{\mathrm{o}}\right)$ em regiões com escassez de dados: estudo de caso no Sul de Minas Gerais. Ambi-Agua, Taubaté, v. 7, n. 2, p. 179-194, 2012. (http://dx.doi.org/10.4136/ambi-agua.763)

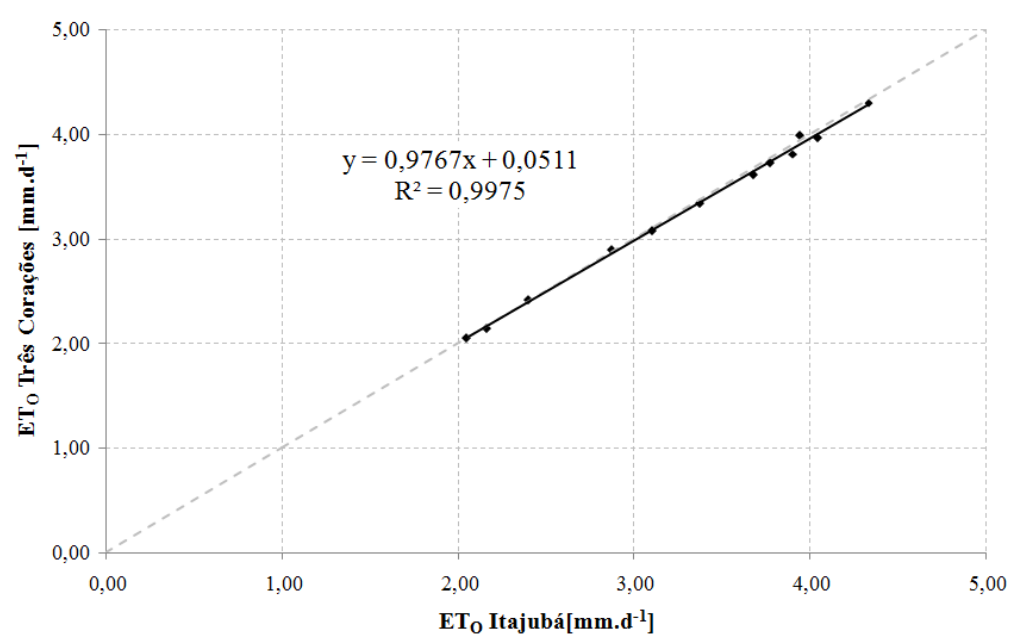

Figura 1. Regressão linear entre as evapotranspirações de referência médias mensais da estação Três Corações e a estação Itajubá.

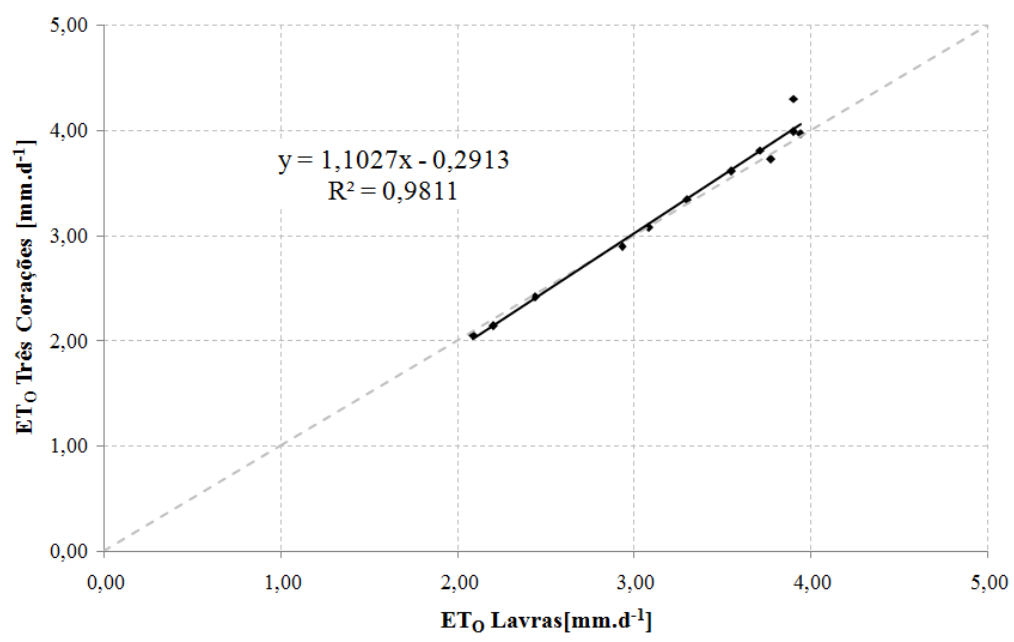

Figura 2. Regressão linear entre as evapotranspirações de referência médias mensais da estação Três Corações e a estação Lavras.

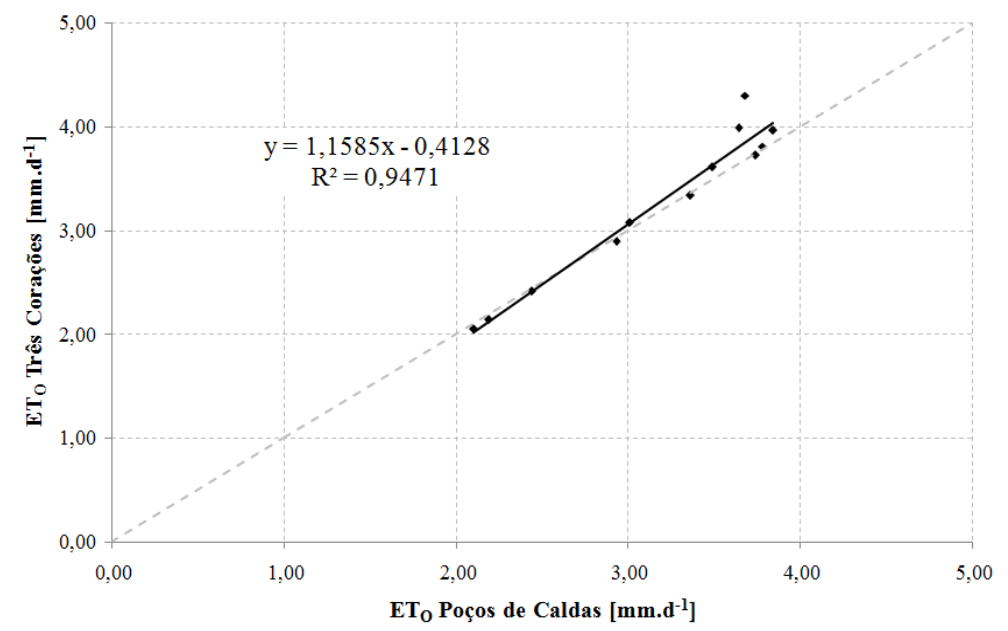

Figura 3. Regressão linear entre as evapotranspirações de referência médias mensais da estação Três Corações e a estação Poços de Caldas. 
Os $\mathrm{R}^{2}$ obtidos nas regressões das Figuras 1 a 3, superiores a 0,9000, demonstram que as ETo médias mensais calculadas para a estação de Três Corações variam de forma proporcional àquelas calculadas para as estações Itajubá, Lavras e Poços de Caldas, representando de forma satisfatória a variabilidade sazonal dos parâmetros meteorológicos utilizados no cálculo, o que corrobora os critérios utilizados na seleção destas estações para a krigagem.

As Figuras 4 e 5 apresentam os mapas de $\mathrm{ET}_{\mathrm{o}}$ médias mensais obtidos por krigagem.

Os mapas apresentados nas Figuras 4 e 5 ilustram a variabilidade espacial e temporal da $\mathrm{ET}_{\mathrm{o}}$ dentro da área de estudo, ao longo das estações do ano. Percebe-se que as menores $\mathrm{ET}_{\mathrm{o}}$, nos meses de janeiro a abril (verão e início de outono), ocorrem a noroeste. Entre maio e agosto (outono e inverno) ocorre uma clara inversão de padrão, com as menores $\mathrm{ET}_{\mathrm{o}}$ sendo identificadas a sudeste. Entre setembro e dezembro (primavera e início de verão), ocorre uma maior variabilidade da $\mathrm{ET}_{\mathrm{o}}$ média mensal, cujos menores valores oscilam entre nordeste e noroeste.

A Tabela 6 relaciona as ETo médias mensais calculadas e interpoladas para a estação do município de Três Corações:

Tabela 6. Evapotranspirações de referência (ETo) médias mensais calculadas e interpoladas para a estação Três Corações.

\begin{tabular}{l|cc|c}
\hline \multicolumn{1}{c|}{ Mês } & $\begin{array}{r}\text { ETo calculada } \\
\text { mm.d }^{-\mathbf{1}}\end{array}$ & $\begin{array}{c}\text { ETo interpolada } \\
\text { mm.d }^{-1}\end{array}$ & $\begin{array}{c}\text { Erro } \\
\text { mm.d }^{-1}\end{array}$ \\
\hline Janeiro & 4,30 & 4,03 & $-0,27$ \\
Fevereiro & 3,99 & 3,89 & $-0,10$ \\
Março & 3,61 & 3,59 & $-0,02$ \\
Abril & 3,08 & 3,08 & 0,00 \\
Maio & 2,41 & 2,42 & 0,01 \\
Junho & 2,05 & 2,07 & 0,02 \\
Julho & 2,14 & 2,18 & 0,04 \\
Agosto & 2,89 & 2,91 & 0,02 \\
Setembro & 3,34 & 3,33 & $-0,01$ \\
Outubro & 3,72 & 3,77 & 0,05 \\
Novembro & 3,96 & 3,98 & 0,02 \\
Dezembro & 3,81 & 3,78 & $-0,03$ \\
\hline
\end{tabular}


VILANOVA, M. R. N.; SIMÕES, S. J. C.; TRANNIN, I. C, B. Interpolação geoespacial da evapotranspiração de referência $\left(\mathrm{ET}_{\mathrm{o}}\right)$ em regiões com escassez de dados: estudo de caso no Sul de Minas Gerais. Ambi-Agua, Taubaté, v. 7, n. 2, p. 179-194, 2012. (http://dx.doi.org/10.4136/ambi-agua.763)
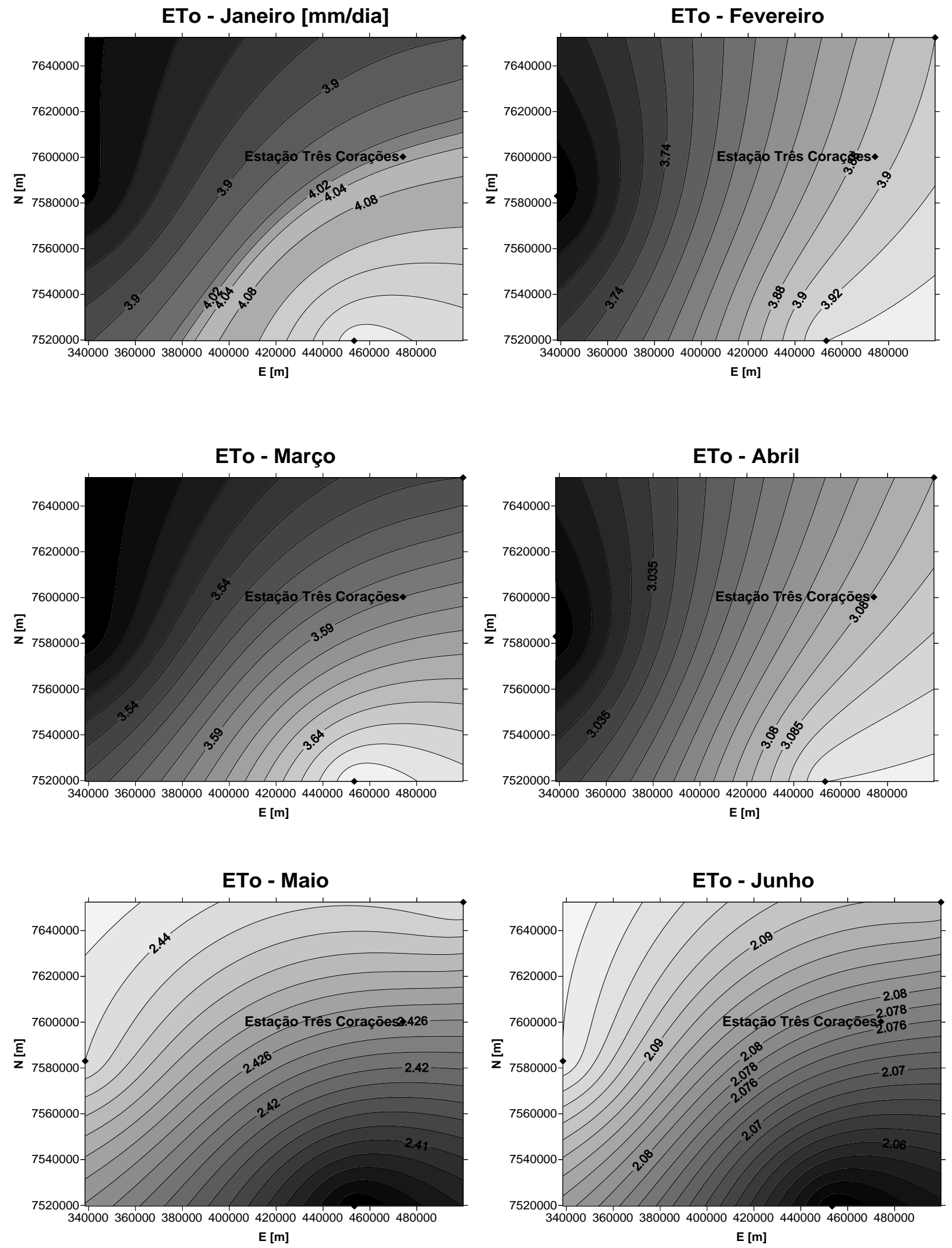

Figura 4. Evapotranspiração de referência média mensal interpolada por krigagem para os meses de janeiro a junho (1975 a 2000). 
VILANOVA, M. R. N.; SIMÕES, S. J. C.; TRANNIN, I. C, B. Interpolação geoespacial da evapotranspiração de referência $\left(\mathrm{ET}_{\mathrm{o}}\right)$ em regiões com escassez de dados: estudo de caso no Sul de Minas Gerais. Ambi-Agua, Taubaté, v. 7, n. 2, p. 179-194, 2012. (http://dx.doi.org/10.4136/ambi-agua.763)
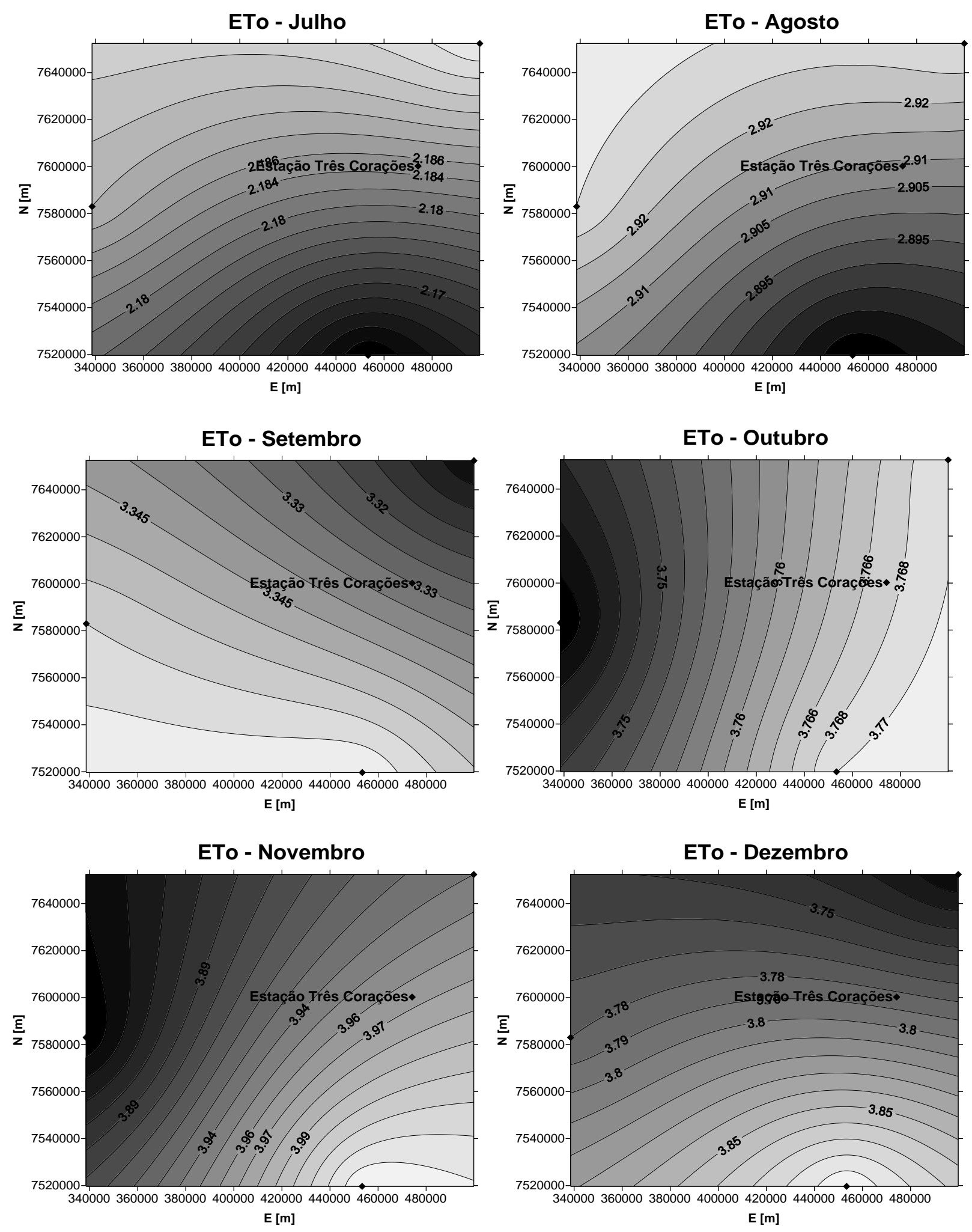

Figura 5. Evapotranspiração de referência média mensal interpolada por krigagem para os meses de julho a dezembro (1975 a 2000).

$\mathrm{Na}$ Tabela 6, percebe-se que os erros entre as $\mathrm{ET}_{\mathrm{o}}$ médias mensais calculadas e interpoladas foram pequenos, na sua maioria inferiores a $2 \%$, com exceção dos meses de janeiro e fevereiro (verão), que apresentaram, respectivamente, $6,28 \%$ e $2,51 \%$. Os menores erros, por sua vez, ocorreram em abril e setembro (outono e primavera). 
A Figura 6 correlaciona os dados calculados e interpolados para a estação de referência, e apresenta os valores dos coeficientes utilizados na análise:

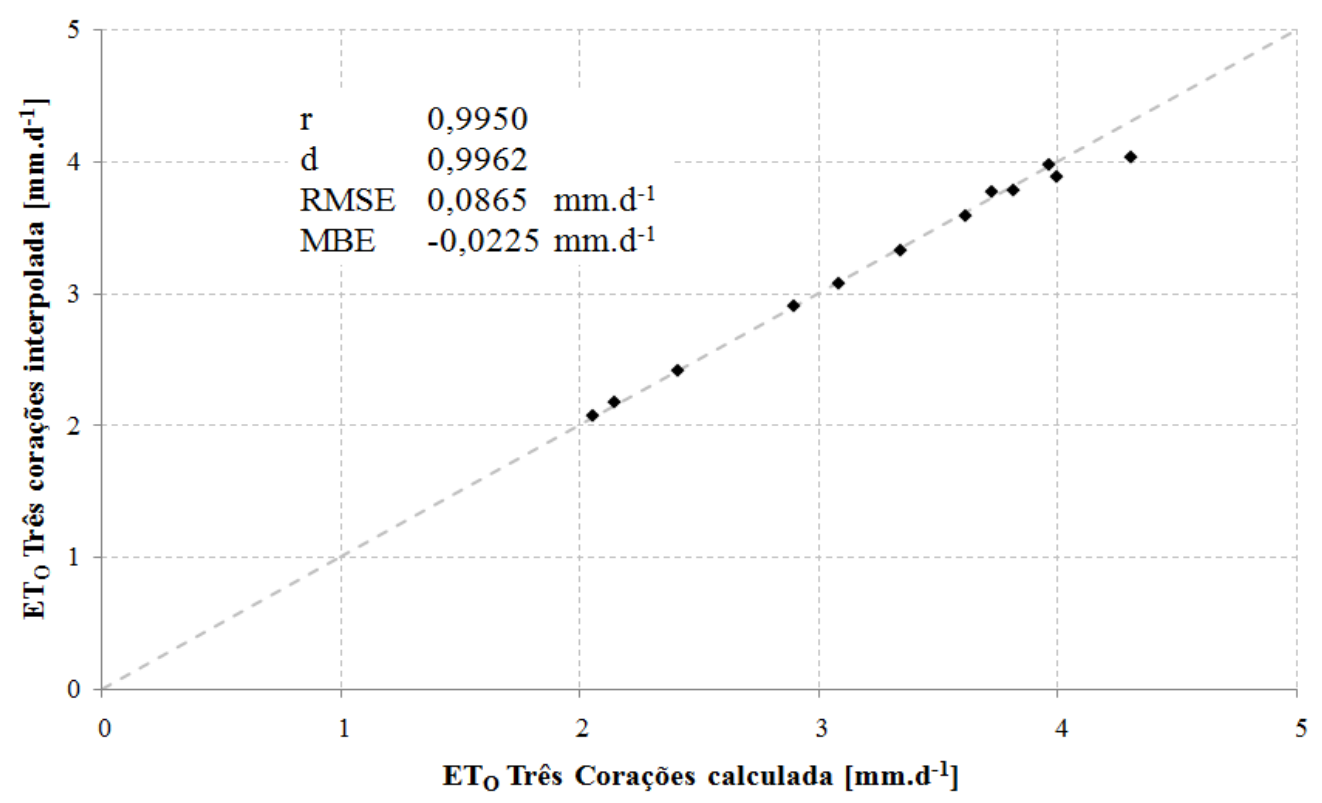

Figura 6. Evapotranspirações de referência médias mensais calculadas e interpoladas para a estação Três Corações.

$\mathrm{O}$ valor de r $(0,9959)$ indica que o modelo de krigagem resultou em $\mathrm{ET}_{\mathrm{O}}$ interpoladas com alta correlação linear em relação aos valores calculados para a estação Três Corações. $\mathrm{O}$ elevado valor do índice d $(0,9962)$, próximo a 1,0000, indica que os valores de $\mathrm{ET}_{\mathrm{o}}$ calculados foram estimados com elevada exatidão pelos dados interpolados espacialmente.

O modelo de interpolação apresentou bom desempenho em relação aos erros de estimativa, como demonstra o RMSE de $0,0865 \mathrm{~mm} \cdot \mathrm{d}^{-1}$. O valor do MBE $\left(-0,0225 \mathrm{~mm} \cdot \mathrm{d}^{-1}\right)$ demonstra que as $\mathrm{ET}_{\mathrm{O}}$ interpoladas apresentam uma pequena sub-estimação em relação aos valores calculados.

A aplicação do $t$-test resultou em $\mathrm{t}=0,0748$ e $\mathrm{P}=0,941$, o que permite concluir que não há diferença estatística relevante entre as $\mathrm{ET}_{\mathrm{o}}$ calculadas e interpoladas para a estação Três Corações. A pequena diferença entre as médias dos dois grupos pode ser atribuída a aleatoriedades, por exemplo, no processo de amostragem dos parâmetros meteorológicos.

Os resultados indicam que a krigagem é uma técnica geoestatística válida para a espacialização da $\mathrm{ET}_{\mathrm{o}}$, o que condiz com os trabalhos de Mello et al. (2003), Markidis et al. (2005), Lemos Filho et al. (2010) e Sartori et al. (2010). A técnica produziu bons resultados durante a espacialização da evapotranspiração de referência, mesmo diante da pequena quantidade de dados (estações) utilizados, o que indica sua aplicabilidade para geração de mapas de $\mathrm{ET}_{\mathrm{o}}$ em regiões com poucas estações/dados hidrometeorológicos. Essa aplicação deve ser realizada de forma criteriosa, utilizando-se ferramentas para a validação dos resultados, como por exemplo, a consideração de uma estação base, conforme realizado nesse trabalho.

Os critérios de seleção das estações para interpolação por krigagem - pequena distância geográfica e inexistência de feições topográficas significativa - atingiram os objetivos esperados, resultando em boa correlação entre as $\mathrm{ET}_{\mathrm{o}}$ calculadas nas estações de interpolação e a estação de referência.

Os resultados também sugerem que a abordagem “calcular e interpolar", menos laboriosa que a "interpolar e calcular", pode ser priorizada em estudos e projetos que exijam a 
interpolação da $\mathrm{ET}_{\mathrm{O}}$, com exceção de casos que exijam, também, a espacialização das variáveis hidrometeorológicas utilizadas no cálculo da $\mathrm{ET}_{\mathrm{O}}$.

\section{CONCLUSÃO}

A krigagem pode ser aplicada para elaboração de mapas de $\mathrm{ET}_{\mathrm{O}}$ em regiões com escassez de dados hidrometeorológicos, desde que procedimentos para seleção das estações meteorológicas utilizadas e validação dos resultados sejam considerados.

\section{AGRADECIMENTOS} trabalho.

Os autores agradecem aos avaliadores do artigo pelas sugestões para aperfeiçoamento do

\section{REFERÊNCIAS}

ALLEN, R. G.; PEREIRA, L. S.; RAES, D.; SMITH, M. Crop evapotranspiration: Guidelines for computing crop water requirements. Roma: FAO, 1998. (FAO Irrigation and drainage paper, 56).

ALVES, M.; BOTELHO, S. A.; PINTO, L. V. A.; POZZA, E. A.; OLIVEIRA, M. S.; FERREIRA, E.; ANDRADE, H. Variabilidade espacial de variáveis geobiofísicas nas nascentes da bacia hidrográfica do Ribeirão Santa Cruz. Revista Brasileira de Engenharia Agrícola e Ambiental, Campina Grande, v. 12, n. 5, p. 527-535, 2008. http://dx.doi.org/10.1590/S1415-43662008000500014

BARGAOUI, Z. K.; CHEBBI, A. Comparison of two kriging interpolation methods applied to spatiotemporal rainfall. Journal of Hydrology, Amsterdã, v. 365, n. 1/2, p. 56-73, 2009. http://dx.doi.org/10.1016/j.jhydrol.2008.11.025

BERnARDO, S.; SOARES, A. A.; MANTOVANI, E. C. Manual de irrigação. 8. ed. Viçosa: Editora UFV, 2008.

BURROUGH, P. A.; MCDONNELL, R. A. Principles of geographical information systems. Oxford: Oxford University Press, 1998.

CÂMARA, G.; MEDEIROS, J. S. (Org.). Geoprocessamento para projetos ambientais. 2. ed. São José dos Campos: INPE, 1998.

CHILDS, C. Interpolating surfaces in ArcGIS Spatial Analyst. ArcUser, Redlands, p. 32-35, jul./set. 2004.

DECOURSEY, W. J. Statistics and probability for engineering applications: with Microsoft ${ }^{\circledR}$ Excel. Woburn: Newness, 2003.

ER-RAKI, S.; CHEHBOUNI, A.; KHABBA, S.; SIMONNEAUZ, V.; JARLAN, L.; OULDBBA, A. et al. Assessment of reference evapotranspiration methods in semi-arid regions: can weather forecast data be used as alternate of ground meteorological parameters? Journal of Arid Environments, Amsterdã, v. 74, n. 12, p. 1587-1596, 2010. http://dx.doi.org/10.1016/j.jaridenv.2010.07.002

FOOD AND AGRICULTURE ORGANIZATION OF THE UNITED NATIONS - FAO. Climwat 2.0. Roma, 2006. 
GUEDES, L. P. C. Otimização de amostragem espacial. 2008. 143f. Tese (Doutorado em Agronomia) - Escola Superior de Agricultura Luiz de Queiroz, Universidade de São Paulo, Piracicaba, 2008.

JACOVIDES, C. P.; KONTOYIANNIS, H. Statistical procedures for the evaluation of evapotranspiration computing models. Agricultural Water Management, Amsterdã, v. 27, n. 3/4, p. 365-371, 1995. http://dx.doi.org/10.1016/0378-3774(95)01152-9

JOURNEL, A. G. Fundamentals of geostatistics in five lessons. Washington: American Geophysical Union, 1989. http://dx.doi.org/10.1029/SC008

LEMOS FILHO, L. C. A.; MELlO, C. R.; FARIA, M. A.; CARVALHO, L. G. Spatialtemporal analysis of water requirements of coffee crop in Minas Gerais State, Brazil. Revista Brasileira de Engenharia Agrícola e Ambiental, Campina Grande, v. 14, n. 2, fev. 2010. http://dx.doi.org/10.1590/S1415-43662010000200007

MARDIKIS, M. G.; KALIVAS, D. P.; KOLLIAS, V. J. Comparison of interpolation methods for the prediction of reference evapotranspiration - An application in Greece. Water Resources Management, [S. 1.], v. 19, n. 3, p. 251-278, 2005. http://dx.doi.org/ $10.1007 / \mathrm{s} 11269-005-3179-2$

MCVICAR, T. R.; VAN NIEL, T. G.; LI, L.; HUTCHINSON, M. F.; MU, X.; LIU, Z. Spatially distributing monthly reference evapotranspiration and pan evaporation considering topographic influences. Journal of Hydrology, Amsterdã, v. 338, p. 196220, 2007. http://dx.doi.org/10.1016/j.jhydrol.2007.02.018

MELlO, C. R.; LIMA, J. M.; SILVA, A. M.; MELlO, J. M.; OLIVEIRA, M. S. Krigagem e inverso do quadrado da distância para interpolação dos parâmetros da equação de chuvas intensas. Revista Brasileira de Ciência Solo, Viçosa, v. 27, n. 5, p. 925933, out. 2003. http://dx.doi.org/10.1590/S0100-06832003000500017

MOTOMIYA, A. V. A.; CORA, J. E.; PEREIRA, G. T. Uso da krigagem indicatriz na avaliação de indicadores de fertilidade do solo. Revista Brasileira de Ciência Solo, Viçosa, v. 30, n. 3, p. 485-496, 2006. http://dx.doi.org/10.1590/S010006832006000300010

ROSS, S. M. Introduction to probability and statistics for engineers and scientists. Burlington: Elsevier Academic Press, 2004.

SARTORI, A. A. C.; SILVA, A. F.; RAMOS, C. M. C.; ZIMBACK, C. R. L. Variabilidade temporal e mapeamento de dados climáticos de Botucatu-SP. Irriga, Botucatu, v. 15, n. 2, p. 131-139, abr. 2010.

SILVA, D.; MEZA, F. J.; VARAS, E. Estimating reference evapotranspiration $\left(\mathrm{ET}_{\mathrm{o}}\right)$ using numerical weather forecast data in central Chile. Journal of Hydrology, Amsterdã, v. 382, p. 64-71, 2010. http://dx.doi.org/10.1016/j.jhydrol.2009.12.018

TOUTENBURG, H. Statistical analysis of designed experiments. 2. ed. Nova Iorque: Springer, 2002.

WILLMOTT, C. J. On the validation of models. Physical Geography, v. 2, n. 2, p. 184-194, 1981. 
VILANOVA, M. R. N.; SIMÕES, S. J. C.; TRANNIN, I. C, B. Interpolação geoespacial da evapotranspiração de referência $\left(E_{\mathrm{o}}\right.$ ) em regiões com escassez de dados: estudo de caso no Sul de Minas Gerais. Ambi-Agua, Taubaté, v. 7, n. 2, p. 179-194, 2012. (http://dx.doi.org/10.4136/ambi-agua.763)

YIN, Y.; WU, S.; ZHENG, D.; YANG, Q. Radiation calibration of FAO56 Penman-Monteith model to estimate reference crop evapotranspiration in China. Agricultural Water Management, Amsterdã, v. 95, n. 1, p. 77-84, 2008. http://dx.doi.org/10.1016/j.agwat. 2007.09.002

ZHANG, X.; KANG, S.; ZHANG, L.; LIU, J. Spatial variation of climatology monthly crop reference evapotranspiration and sensitivity coefficients in Shiyang river basin of northwest China. Agricultural Water Management, Amsterdã, v. 97, n. 10, p. 15061516, 2010. http://dx.doi.org/10.1016/j.agwat.2010.05.004 